"Effect of base stacking on the relative thermodynamic stability of oligonucleotide complexes: A spectroscopic study" Murphy, D., Doyle, H., Eritja, R., Redmond, G.

J. Biomol. Struct. Dyn., 22(2), 195-204 (2004).

\title{
Effect of Base Stacking on the Relative Thermodynamic Stability of Oligonucleotide Complexes: A Spectroscopic Study.
}

Deirdre Murphy, $\dagger$ Hugh Doyle, $\dagger$ Ramon Eritja $\neq$ and Gareth Redmond*, $†$.

$\dagger$ Nanotechnology Group, NMRC, Lee Maltings, Cork, Ireland, and $\ddagger$ Department of Structural

Biology, Instituto de Biología Molecular de Barcelona, C.S.I.C., Jordi Girona 18-26, E-08034

Barcelona, Spain.

* To whom correspondence should be addressed at: Nanotechnology Group, NMRC, Lee Maltings, Cork, Ireland. E-mail: gareth.redmond@nmrc.ie. 


\begin{abstract}
Three-strand oligonucleotide complexes are employed to assess the effect of base stacking and base pair mismatch on the relative thermodynamic stabilities of oligonucleotide duplexes. The melting behaviour of three-strand oligonucleotide complexes incorporating nicks and gaps as well as internal single base mismatches is monitored using temperature-dependent optical absorption spectroscopy. A sequential three-state equilibrium model is used to analyse the measured melting profiles and evaluate thermodynamic parameters associated with dissociation of the complexes. The free-energy of stabilisation of a nick complex compared to a gap complex due to base stacking is determined to be $-1.9 \mathrm{kcal} / \mathrm{mol}$. The influence of a mispaired base in these systems is shown to destabilise a nick complex by 3.1 $\mathrm{kcal} / \mathrm{mol}$ and a gap complex by $2.8 \mathrm{kcal} / \mathrm{mol}$, respectively.
\end{abstract}




\section{Introduction}

DNA microarrays are versatile tools employed for gene expression analysis, gene profiling, DNA sequencing and mutation detection. Numerous methods have been developed to enhance the performance of microarrays for applications such as, e.g., sequencing by hybridisation. One method in particular, which has yielded demonstrable improvements in this regard, is contiguous stacking hybridisation (CSH), first reported by Lysov et al. in 1988 (1). Stacking hybridisation occurs when two or more short oligonucleotides hybridise to a long oligonucleotide in a contiguous tandem manner. The point at which the two short oligonucleotides meet is without a connecting covalent bond and is known as a nick. At this point a coaxial base stacking interaction occurs between the terminal bases of the two short oligonucleotides, which increases the stability of the short strands and makes them more difficult to dissociate than the equivalent sequences hybridised in a non-contiguous manner. $\mathrm{CSH}$ can increase the efficiency of sequencing by hybridisation on microarrays by enabling reductions in the size of an oligonucleotide matrix (2-5). For example, the efficiency of a microarray containing $4^{(\mathrm{n}+5)}(\mathrm{n}+5)$ mers or $4^{(\mathrm{n}+10)}(\mathrm{n}+10)$ mers may be achieved using a microarray containing only $4^{\mathrm{n}}$ nmers (5).

The stabilising effect of coaxial base stacking may also be utilised to improve the resolution of microarray technologies for mutation detection applications (6-9). For example, a duplex of a long and short strand, one of which is tethered to a surface, can be used to capture a single target strand from solution whereby the stacking interaction between the duplex and the target provides enhanced stringency in distinguishing between different target sequences (8). In this manner, Mirzabekov and co-workers have shown that contiguously stacked 5mers are more sensitive to the presence of mismatches in their duplexes than longer probes and, using this approach, have developed several novel CSH-based mutation detection strategies (6-8). 
Coaxial stacking has also proven useful in other applications such as the improvement of nucleic acid cleavage rates (10), and the investigation of RNA folding (11). A comparative understanding of DNA sequence-dependent thermodynamic stability and solution hybridisation behaviour would have direct practical applications in the design of multiplex reactions (12). In this regard, co-operative parameter interactions at the nick position have previously been calculated using a complementary addressed modification titration (CAMT) method (13-15). Free energy values of coaxial stacking for a range of possible interaction types at a nick position have also been determined for two-strand systems where a short strand contiguously stacks to a hairpin oligonucleotide $(16,19)$. To extend these studies, the thermodynamic properties of three component complexes comprising a duplex formed between a long strand and two contiguously hybridised shorter strands are investigated in this work. The thermodynamic stabilisation provided by coaxial stacking at the nick position in these systems is estimated. Finally the effect of inserting a mismatched base pair into such systems is also determined. The three-component complexes studied here are being extensively used in sequecing by hybridisation (1-5) and mismatch detection using oligonucleotide-gold nanoparticles (20-23). The data presented here is specially relevant for the design of the oligonucleotide probes used in these systems.

\section{Experimental}

\section{Oligonucleotide Design}

In this paper, the effect of base stacking and base pair mismatch on the solution thermodynamics of model three-strand oligonucleotide complexes is assessed. To this end, a range of oligonucleotide sequences was designed; see Figure 1. Figure 1(a) shows the nick complex, a three-strand duplex where two 12mer oligonucleotide strands, $\mathrm{X}$ and $\mathrm{Y}$, are hybridised to a 24 mer oligonucleotide strand, $\mathrm{Z}$, in a contiguous fashion. The point at which 
the two short oligonucleotides meet is called a nick. It is at this point that a 5' $\mathrm{G}-\mathrm{C} 3$ ' base stacking interaction will occur even though the two short strands are not covalently linked. Note also that all sequences contain terminal GC base pairs to minimise fraying of the helix at the junction of the 12 mer sequences and to thereby enhance the magnitude of the base stacking effect (17). Figure 1(b) shows the gap complex. This duplex has the same two X and Y 12mer strands, but in this case they are hybridised in a non-contiguous manner to a longer 26mer strand, Z'. The two 12 mer strands are separated by the two A bases inserted into the longer strand (underlined) and hence should not exhibit the extra stability afforded to the nick complex by base stacking. Figure 1(c) shows the nick/mismatch complex. This duplex is analogous to the nick complex except there is a base pair mismatch between the 12 mer $Y^{\prime}$ and the 24 mer $\mathrm{Z}$ strands. The position at which this $\mathrm{G} \cdot \mathrm{G}$ mismatch occurs is underlined. Figure 1(d) shows the gap/mismatch complex, which comprises the longer 26mer Z'" strand, the X strand and the mismatch bearing Y' strand.

\section{Materials}

The following materials were obtained from Sigma-Aldrich (UK): NaH2PO4, Na2HPO4 and $\mathrm{NaCl}$. All oligonucleotides were synthesized by MWG Biotech (Germany) on a $0.2 \mu \mathrm{mol}$ scale. All experiments used nanopure $\mathrm{H} 2 \mathrm{O}$ (18.2 M $\Omega$ ) purified using an Elgastat Prima purification system.

\section{Methods}

Before using the oligonucleotides, a working stock solution was prepared in order to minimise thawing and freezing of a single solution and also to reduce pipetting error. The oligonucleotides were prepared for melting experiments by dissolving them in melting buffer (0.3 M NaCl, $10 \mathrm{mM}$ Phosphate buffer, $\mathrm{pH}$ 7). The four three-strand complexes that were studied were prepared by mixing equimolar amounts of the $\mathrm{X}, \mathrm{Y}$ or $\mathrm{Y}^{\prime} 12 \mathrm{mer}$ strands with appropriate complementary 24 mer or $26 \mathrm{mer}, \mathrm{Z}$ or Z', strands. The solutions were heated to 
$90{ }^{\circ} \mathrm{C}$ for $5 \mathrm{~min}$ and allowed to cool to room temperature and hybridise for $2 \mathrm{~h}$. The total concentration of the strands used in each melting measurement was $4.3 \mu \mathrm{mol}$.

The sample mixture was placed in a stoppered 1-cm path-length cuvette and UV-visible optical absorption spectra were recorded at $2{ }^{\circ} \mathrm{C}$ intervals while the sample was heated from $20-90{ }^{\circ} \mathrm{C}$. The sample was allowed $2 \mathrm{~min}$ between each reading to reach the next temperature and stabilise at that temperature. Melting curves were collected on a Shimadzu 2401 double beam spectrophotometer equipped with a Shimadzu constant temperature cell holder attached to a Grant W6-KD precision thermostatic circulator. The absorbance of the buffer alone was recorded in the reference slot of the double beam spectrophotometer and automatically subtracted from the oligonucleotide reaction absorbance data. The linear temperature offset $\left(2-4^{\circ} \mathrm{C}\right)$ between the sample mixture in the reaction cuvette and the water in the water bath was independently determined using a calibrated thermocouple and a suitable correction was applied to the measured data. Data were collected as wavelength versus absorbance spectra and plots of absorbance versus temperature at $260 \mathrm{~nm}$ were then prepared. At least two melting experiments were carried out for each of the complexes. Melting experiments were conducted for each of the duplex components, $\mathrm{XZ}$ or $\mathrm{XZ}$ ', under identical experimental conditions to those employed for the three-strand complexes.

\section{Results}

Experimentally obtained absorption versus temperature melting curves for each of the four complexes studied are shown in Figure 2. Two distinct melting transitions may be distinguished on each plot corresponding to two melting events. This is expected as these complexes were designed such that the two short 12 mer strands, $\mathrm{X}$ and $\mathrm{Y}$, would melt from the longer 24 mer or 26 mer, $\mathrm{Z}$ or Z', strands at different temperatures. The separation of the melting temperatures of the 12 mers was achieved by designing one of the 12 mer strands, $\mathrm{X}$, with a higher GC content and the other 12 mer strands, Y or Y', with a higher AT content; see 
Figure 1. It is well known that DNA duplex stability depends on percent GC content (18). As such, the $12 \mathrm{mer}, \mathrm{X}$, is expected to melt at a higher temperature due to the greater stability of GC base pairs versus AT base pairs. Similar studies carried out by Benight et al. (19) employed a hairpin to ensure that a portion of the duplex would melt at higher temperature.

The lower temperature transitions on the melting curves (from 10 to $c a .45{ }^{\circ} \mathrm{C}$ ) reflect the dissociation of the $\mathrm{Y}$ or $\mathrm{Y}^{\prime}$ strands from the three-strand complexes. The second transition (from $c a .45$ to $90{ }^{\circ} \mathrm{C}$ ) reflects the subsequent dissociation of the $\mathrm{X}$ strand from the remaining $\mathrm{XZ}$ or XZ" duplexes. The data of Figure 2 indicates that the $\mathrm{XZ}$ and $\mathrm{XZ}$ "' melting behaviour is almost identical for each of the complexes. It can therefore be assumed that melting of either the $\mathrm{XZ}$ or $\mathrm{XZ}$ ', duplexes is independent of the presence of the Y or Y' strands. It can also be seen, in Figure 2, that the dissociation behaviour of the $\mathrm{Y}$ or $\mathrm{Y}$ ' differs for each complex. Thus dissociation of the $\mathrm{Y}$ or $\mathrm{Y}^{\prime}$ strand from a complex is affected both by the interruption of base stacking caused by the inserted gap and by the presence of the mismatch.

To quantitatively analyse the dissociation behaviour of the $\mathrm{Y}$ or $\mathrm{Y}^{\prime} 12 \mathrm{mers}$ in response to such sequence changes for the four complexes, melting profiles were determined for the duplexes which formed when the 12 mer X hybridised to either the 24 mer Z or the 26 mer Z' at the same concentration and under the same solution conditions employed for the threestrand complexes. Figure 3 shows the melting profiles for each of the three-strand complexes (data of Figure 2), with insets showing the respective melting curves for each of the $\mathrm{XZ}$ or XZ' duplex components alone. This data is used in the next section to deconvolute the normalised melting curves of the YZ, YZ', Y'Z and Y'Z', duplexes from the three-strand complex melting profiles of Figure 3. 


\section{Data Analysis}

The melting data for each of the complexes were analysed in terms of a sequential three state equilibrium model. Using this approach, melting of, e.g., the nick complex, XZY, may be represented as follows:

$$
\mathrm{XZY} \leftrightarrow \mathrm{XZ}+\mathrm{Y} \leftrightarrow \mathrm{X}+\mathrm{Z}+\mathrm{Y} \quad \text { Equation } 1
$$

The first equilibrium process corresponds to dissociation of the $12 \mathrm{mer} \mathrm{Y}$ strand from the three-strand XZY complex. The second equilibrium occurs at a higher temperature and corresponds to dissociation of $\mathrm{X}$ and $\mathrm{Z}$. Following this model, the melting curve for the first step, i.e., the melting of the $12 \mathrm{mer} \mathrm{Y}$ from the three-strand complex, can be extracted from the measured data by analysis of the melting profiles of the complex in conjunction with the melting profile of the corresponding $\mathrm{XZ}$ duplex alone. This analytical approach has previously been employed to extract melting profiles of DNA triplexes (24) and, also, of three-strand DNA complexes incorporating hairpins (19).

To this end, the contribution of the $\mathrm{XZ}$ duplex melting signal to the complex melting signal is first evaluated. The XZ melting curve is shown as an inset to Figure 3(a). The upper $\left(\mathrm{A}_{\mathrm{U}} \mathrm{XZ}\right.$ versus $\mathrm{T})$ and lower $\left(\mathrm{A}_{\mathrm{L}}{ }^{\mathrm{XZ}}\right.$ versus $\left.\mathrm{T}\right)$ baselines of the $\mathrm{XZ}$ melting curve are determined from linear fits of the sloping regions before and after the main melting transition. Equation 2 below is then employed to determine the fraction of melted duplex $\mathrm{XZ}$ present as a function of temperature, i.e., $\theta_{\mathrm{XZ}}(\mathrm{T})$, by normalising the experimental $\mathrm{XZ}$ melting curve between the upper and lower baseline fits:

$$
\theta_{X Z}=\frac{A^{X Z}-A_{L}^{X z}}{A_{U}^{X z}-A_{L}^{X z}} \quad \text { Equation 2 }
$$


where $\mathrm{A}^{\mathrm{XZ}}$ represents the absorbance at $260 \mathrm{~nm}$ versus temperature values that were collected during the $\mathrm{XZ}$ melting experiment, $\mathrm{A}_{\mathrm{U}} \mathrm{XZ}$ are the upper baseline values of the $\mathrm{XZ}$ melting transition and $\mathrm{A}_{\mathrm{L}}{ }^{\mathrm{XZ}}$ are the lower baseline values of the $\mathrm{XZ}$ melting transition.

Next, the middle $\left(\mathrm{A}_{\mathrm{M}}{ }^{\mathrm{XZY}}\right.$ versus $\left.\mathrm{T}\right)$ and lower $\left(\mathrm{A}_{\mathrm{L}}{ }^{\mathrm{XZY}}\right.$ versus $\left.\mathrm{T}\right)$ baselines of the first $\mathrm{XZY}$ melting transition are determined from linear fits of the sloping regions before and after this transition on the XZY complex melting curve shown in Figure 3(a). The fraction of 12mer Y dissociated from the XZY complex at each given temperature, $f_{D}$, is then obtained from the measured XZY melting profile using the following expression (19):

$$
f_{D}=\frac{A^{X Z Y}-A_{L}^{X Z Y}}{A_{M}^{X Z Y}-A_{L}^{X Z Y}+\theta_{X Z}\left(A_{U}^{X Z}-A_{L}^{X Z}\right)} \quad \text { Equation 3 }
$$

An $f_{D}$ versus temperature, $T$, curve therefore reflects only the specific dissociation of the $Y$ strand from the XZY complex. Performing a similar analysis for the gap, nick/mismatch and gap/mismatch complexes yields a series of $f_{D}$ versus $T$ curves for each of the four complexes; see Figure 4.

As the data treatment described above explicitly removes the contribution of $\mathrm{XZ}$ melting from the measured XZY complex melting curve, the nick $f_{D}$ versus $T$ curve reflects the single bimolecular reaction that is the melting of $\mathrm{XZY}$ complexes producing $\mathrm{XZ}$ duplexes and $\mathrm{Y}$ 12 mers. The observed equilibrium constant, $\mathrm{K}_{\mathrm{obs}}$, for this first transition is:

$$
\mathrm{K}_{\mathrm{obs}}=\frac{[\mathrm{XZ}][\mathrm{Y}]}{[\mathrm{XZY}]}
$$

Equation 4

where $[\mathrm{XZ}],[\mathrm{Y}]$ and $[\mathrm{XZY}]$ are the concentrations of the strands $\mathrm{XZ}, \mathrm{Y}$ and $\mathrm{XZY}$, respectively. Also, the total concentration of the strands, $\mathrm{C}_{\mathrm{T}}$ is: 


$$
\mathrm{C}_{\mathrm{T}}=2[\mathrm{XZY}]+[\mathrm{XZ}]+[\mathrm{Y}] \quad \text { Equation } 5
$$

To evaluate quantitative thermodynamic parameters associated with this melting step, an analytical expression was derived and employed to relate $f_{D}$ to $K_{o b s}$ and $C_{T}$ as follows (19):

$$
f_{D}=\frac{K_{o b s}\left(\sqrt{1+2 C_{T} / K_{o b s}}-1\right)}{C_{T}} \quad \text { Equation } 6
$$

As $\mathrm{K}_{\mathrm{obs}}$ is dependent on temperature, Equation 6 is an expression relating $\mathrm{f}_{\mathrm{D}}$ to $\mathrm{T}$ and may therefore be fitted to extract the thermodynamic reaction parameters $\Delta \mathrm{H}^{\circ}$ and $\Delta \mathrm{S}^{\circ}$ from $\mathrm{K}_{\mathrm{obs}}$ via the following relationship:

$$
\mathrm{K}_{\mathrm{obs}}=\exp \left[\left(\mathrm{T} \Delta \mathrm{S}^{\circ}-\Delta \mathrm{H}^{\circ}\right) / \mathrm{RT}\right] \quad \text { Equation } 7
$$

Curve fitting was undertaken using a Levenberg-Marquardt non-linear least-squares fitting program and Mathematica ${ }^{\circledR} 4$ software. The solid lines in Figure 4 show the fits to the data obtained using Equations 6 and 7 above, i.e., with $\Delta \mathrm{H}^{\circ}$ and $\Delta \mathrm{S}^{\circ}$ as adjustable parameters assumed to be independent of temperature. The normalised melting data, $f_{D}$, are displayed using symbols. Fits were deemed acceptable when the evaluated adjustable parameters did not vary by more than $0.1 \%$ between successive iterations. The excellent correlation between the fits and the experimental data is clear, supporting the assumption that the normalised $f_{D}$ data of Figure 4 could reasonably be analysed in terms of a bimolecular interaction. The thermodynamic parameters obtained in this manner for each of the melting reactions are shown in Table I. Gibbs free-energy values for the melting transition of each complex were determined from these $\Delta \mathrm{S}^{\circ}$ and $\Delta \mathrm{H}^{\circ}$ values using the relationship $\Delta \mathrm{G}^{\circ}=\Delta \mathrm{H}^{\circ}-\mathrm{T} \Delta \mathrm{S}^{\circ}$ and are likewise shown. 


\section{Discussion}

The objective of the present paper is to assess the effect of base stacking and base pair mismatch on the relative thermodynamic stabilities of three-strand oligonucleotide complexes. In the preceding section, quantitative estimates of the thermodynamic parameters associated with melting of four such complexes were evaluated by using a three-state sequential equilibrium model to analyse optical melting profiles of each of the complexes. With this in mind, the melting profiles were acquired under identical experimental conditions from samples that contained essentially identical oligonucleotide concentrations. Therefore, while melting profiles of the various complexes were not measured as a function of total strand concentration, the conditions, methods and assumptions employed during the foregoing analysis are considered to provide a suitable basis for comparative assessment of the extracted thermodynamic data.

In this regard, both nick complexes, i.e., nick (XZY) and nick/mismatch (XZY'), were found to exhibit similar melting transition enthalpy and entropy values for melting of their respective $\mathrm{Y}$ and $\mathrm{Y}^{\prime}$ strands. The enthalpy values $\left(20^{\circ} \mathrm{C}\right)$ were $\Delta \mathrm{H}^{\circ}=-94.5 \mathrm{kcal} / \mathrm{mol}$ for the nick complex and $\Delta \mathrm{H}^{\circ}=-93.0 \mathrm{kcal} / \mathrm{mol}$ for the nick/mismatch complex. The corresponding entropy values were $\Delta \mathrm{S}^{\circ}=276.5 \mathrm{cal} / \mathrm{molK}$ and $\Delta \mathrm{S}^{\circ}=282.0 \mathrm{cal} / \mathrm{molK}$, respectively. Also, the two gap complexes, i.e., gap (XZ'YY) and gap/mismatch (XZ''Y'), exhibited similar behaviour with $\Delta \mathrm{H}^{\circ}=-82.9 \mathrm{kcal} / \mathrm{mol}$ and $\Delta \mathrm{S}^{\circ}=243.3 \mathrm{cal} / \mathrm{molK}$ for the gap complex and $\Delta \mathrm{H}^{\circ}$ $=-83.1 \mathrm{kcal} / \mathrm{mol}$ and $\Delta \mathrm{S}^{\circ}=247.5 \mathrm{cal} / \mathrm{molK}$ for the gap $/$ mismatch complex. Estimated uncertainties of $\Delta \mathrm{H}^{\circ}$ and $\Delta \mathrm{S}^{\circ}$ values were $\sim 3 \%$.

From the extracted $\Delta \mathrm{H}^{\circ}$ and $\Delta \mathrm{S}^{\circ}$ values, the free-energy changes associated with these melting transitions were determined; see Table I. For the nick complex, the melting transition free-energy $\left(20^{\circ} \mathrm{C}\right)$ was evaluated at $\Delta \mathrm{G}^{\circ}=-13.5 \pm 0.1 \mathrm{kcal} / \mathrm{mol}$. This value is similar to those calculated using nearest neighbour methods for a $12 \mathrm{mer} \mathrm{Y}$ strand hybridised to a 
complementary sequence of the same length; see Table I $(17,25)$. For the gap complex, $\Delta \mathrm{G}^{\circ}=$ $-11.6 \pm 0.1 \mathrm{kcal} / \mathrm{mol}$. For the nick $/$ mismatch and gap $/$ mismatch complexes, $\Delta \mathrm{G}^{\circ}=-10.4 \pm 0.1$ and $-8.8 \pm 0.1 \mathrm{kcal} / \mathrm{mol}$, respectively.

By careful oligonucleotide design and through application of the analytical approach employed to extract the thermodynamic parameters, contributions to the evaluated transition free-energies arising from melting of the $\mathrm{XZ}$ or $\mathrm{XZ}$ ' components of each complex have been carefully excluded. Consequently, the extracted free-energies for each melting transition may be considered to be the sum of two independent contributions, one for the free-energy of the duplex component under study, i.e., melting of the 12 mer $\mathrm{Y}$ or $\mathrm{Y}^{\prime}$ from $\mathrm{XZ}$ or $\mathrm{XZ}$ ', and one for nick or gap, respectively. The former thermodynamic contribution therefore accounts for all factors pertaining to the formation of this duplex component that do not explicitly involve a nick or a gap and is expected to depend only on the sequence and length of $\mathrm{Y}^{\prime}$ or $\mathrm{Y}^{\prime}$ and $\mathrm{XZ}$ or XZ', and on the ratio of their concentrations.

Comparing nick (XZY) with gap (XZ'Y) complexes, the duplex regions of these complexes are identical in sequence. The free-energy contribution due to melting of the 12 mer $\mathrm{Y}$ from the $\mathrm{XZ}$ or $\mathrm{XZ}$ ' components may thus be assumed to be equal when the concentrations of both $\mathrm{Y}$ and $\mathrm{XZ}$ or XZ', are equal. This was the case during acquisition of the melting data, as described in the Experimental section. As a result, subtraction of the extracted free-energy value of the gap complex from that of the nick complex should allow accurate determination of the relative free-energy difference between this nick and gap, i.e., $\Delta \mathrm{G}^{\circ} \mathrm{XZY}-\Delta \mathrm{G}^{\circ}{ }_{\mathrm{XZ}}{ }^{\prime} \mathrm{Y}=-1.9 \pm 0.2 \mathrm{kcal} / \mathrm{mol}$

The magnitude of the value of the free energy of this nick is likely to be a lower limit since it has been determined relative to a gap complex that has a 3'A-A sequence adjacent to the duplex component under study. It is known that single strand 3' overhangs of this type can stabilise an adjoining duplex (26-28). In particular, it has been shown that a 3'A-A overhang 
adjacent to a $\mathrm{C}$ base on an adjoining duplex, similar to that employed in this study exhibits a stabilisation contribution of $-0.5 \mathrm{kcal} / \mathrm{mol}$ (29). This suggests that the expected stabilisation of the duplex component by the A-A gap employed herein may be $-0.5 \mathrm{kcal} / \mathrm{mol}$ or more, neglecting differences in strand sequence and salt concentration. As a consequence, the likely free-energy of stabilisation due to the nick may actually be $-2.4 \mathrm{kcal} / \mathrm{mol}$.

Comparing nick/mismatch (XZY') with gap/mismatch (XZ'’Y') complexes, while a single base $(\mathrm{G} \cdot \mathrm{G})$ mismatch has been incorporated into each $12 \mathrm{mer} \mathrm{Y}^{\prime}$ strand, the duplex regions of these complexes are also identical in sequence. Further, during the melting experiments, the concentrations of $\mathrm{Y}^{\prime}$ and $\mathrm{XZ}$ or $\mathrm{XZ}$ ' were made equal; see the Experimental section. Therefore, performing a subtraction for these complexes yields a value of the relative freeenergy difference between this nick and gap, i.e., $\Delta \mathrm{G}^{\circ} \mathrm{XZY}^{\prime}-\Delta \mathrm{G}^{\circ} \mathrm{XZ}^{\prime} \mathrm{Y}^{\prime}=-1.6 \pm 0.2 \mathrm{kcal} / \mathrm{mol}$. Following incorporation of the estimated duplex stabilisation effect of the "dangling" A-A gap as above, the free-energy of stabilisation due to the nick in these mismatch-bearing complexes is likely to be $-2.1 \mathrm{kcal} / \mathrm{mol}$. Note also that, for the $\mathrm{XZ}$ '' $\mathrm{Y}^{\prime}$ complex, in this case, it is assumed that the magnitude of the free-energy of stabilisation due to the 3'A-A gap is unperturbed by the presence of the single base mismatch, since the single base $(\mathrm{G} \cdot \mathrm{G})$ mismatch incorporated into the $12 \mathrm{mer} \mathrm{Y}^{\prime}$ strand is located 4 bases from the gap position. It appears, therefore, that both the fully complementary complexes and the complexes that incorporate the G.G mismatch investigated above are stabilised to approximately the same extent by nick formation.

Comparing nick (XZY) with nick/mismatch (XZY') complexes, it is reasonable to expect that the magnitude of the free-energy contribution due to the nick is equivalent for both of these complexes given its position in the $12 \mathrm{mer} \mathrm{Y}^{\prime}$ strand. Again, during the melting experiments, the concentrations of both $\mathrm{Y}$ or $\mathrm{Y}^{\prime}$ and $\mathrm{XZ}$ were made equal. As a result, subtraction of the extracted free-energy value of the nick/mismatch complex from that of the 
nick complex yields the relative free-energy difference due to the presence of the specific $G \cdot G$ mismatch in this nick complex, i.e., $\Delta \mathrm{G}^{\circ} \mathrm{XZY}-\Delta \mathrm{G}^{\circ}{ }_{\mathrm{XZY}}{ }^{\prime}=-3.1 \pm 0.2 \mathrm{kcal} / \mathrm{mol}$. Performing an analogous subtraction for the gap (XZ'YY) and gap/mismatch (XZ''Y') complexes yields a value of the relative free-energy difference due to the presence of this mismatch in this gap complex, i.e., $\Delta \mathrm{G}^{\circ} \mathrm{XZ} \mathrm{X}^{\prime} \mathrm{Y}-\Delta \mathrm{G}^{\circ} \mathrm{XZ}^{\prime} \mathrm{Y}^{\prime}=-2.8 \pm 0.2 \mathrm{kcal} / \mathrm{mol}$. In the latter case, it is assumed that the magnitude of the free-energy of stabilisation due to the presence of the 3'A-A overhang is equivalent for both of the gap complexes, and will therefore cancel during subtraction. The similarity of the two values derived above indicates that these nick and gap complexes are destabilised by almost the same extent following incorporation of the G.G single base mismatch.

\section{Summary}

The objective of this study was to assess the effect of base stacking and base pair mismatch on the relative thermodynamic stabilities of oligonucleotide duplexes. To this end, threestrand oligonucleotide complexes incorporating nicks and gaps as well as internal single base mismatches were designed and synthesised. The melting behaviour of these complexes was monitored using temperature-dependent optical absorption spectroscopy. Analysis of measured melting profiles was performed using a sequential three-state equilibrium model that permitted quantitative evaluation of thermodynamic parameters associated with dissociation of the complexes. The free-energy of stabilisation of a nick complex due to base stacking was determined. While, for the mismatched sequences, both nick and gap complexes were destabilised to the same extent by introduction of the mismatch, the extent of stabilisation due to nick formation was similar to that determined for the fully complementary sequences. The technique described here is therefore generally applicable to the evaluation of key structure- and sequence-dependent thermodynamic quantities that are of importance for the rational optimisation of stacking assisted DNA capture and sequence analysis methods. 


\section{Acknowledgements}

This work was supported by the HEA PRTLI1 Nanoscale Science and Technology Initiative. 


\section{References}

1. Y.P. Lysov, V.L. Florentiev, A. Khorlin, K. Khrapko, V. Shick and A.D. Mirzabekov, Proc. Acad. Sci. USSR 303, 1508-1511 (1988).

2. Y.P. Lysov, A.A. Chernyi, A.A. Balaeff, K.L. Beattie and A.D. Mirzabekov, J. Biomol. Struct. Dyn. 11, 797-812 (1994).

3. S. Parinov, V. Barsky, G. Yershov, E Kirillov, E. Timofeev, A. Belgovskiy and A. Mirzabekov, Nucleic Acids Res. 24, 2998-3004 (1996).

4. N.E. Broude, T. Sano, C.L. Smith and C.R. Cantor, Proc. Natl. Acad. Sci. USA 91, 3072-3076 (1994).

5. A.A. Stomakhin, V.A. Vasiliskov, E. Timofeev, D. Schulga, R.J. Cotter and A.D. Mirzabekov, Nucleic Acids Res. 28, 1193-1198 (2000).

6. G. Yershov, V. Barsky, A. Belgovskiy, E. Kirillov, E. Kreindlin, I. Ivanov, S. Parinov, D. Guschin, A. Drodishev, S. Dubiley and A. Mirzabekov, Proc. Natl. Acad. Sci. USA 93, 4913-4918 (1996).

7. A.V. Fotin, A.L. Drobyshev, D.Y. Proudnikov, A.N. Perov and A.D. Mirzabekov, Nucleic Acids Res. 26, 1515-1521 (1998).

8. V.A. Vasiliskov, D.V. Prokopenko and A.D. Mirzabekov, Nucleic Acids Res. 29, 2303-2313 (2001).

9. R. Maldonado-Rodriguez, M. Espinosa-Lara, P. Loyola-Abitia, W.G. Beattie and K.L. Beattie, Molecular Biotechnology 11, 13-25 (1999).

10. S.B. Lin, K.R. Blake, P.S. Miller and P.O. Ts'o, Biochemistry 28, 1054-1061 (1989).

11. A.E. Walter, D.H. Turner, J. Kim, M.H. Lyttle, P. Muller, D.H. Mathews, and M. Zuker, Proc. Natl. Acad. Sci. USA 91, 9218-9222 (1994).

12. A.S. Benight, P. Pancoska, R. Owczarzy, P.M. Vallone, J. Nesetril and P.V. Riccelli, Methods in Enzymology 340, 165-192 (2001). 
13. A. Adeenah-Zadah, D.G. Knorre and O.S. Fedorova, J. Biomol. Struct. Dyn. 15, 369380 (1997).

14. O.S. Fedorova, A. Adeenah-Zadah, E.V. Bichenkova and D.G. Knorre, J. Biomol. Struct. Dyn. 13, 145-166 (1995).

15. V.V. Koval, N.A. Lokteva, S.L. Karnaukhova and O.S. Fedorova, J. Biomol. Struct. Dyn. 17, 259-265 (1999).

16. D.V. Pyshyi and E.M. Ivanova, Russ. Chem. Bull., Int. Ed. 51, 1145-1155.

17. J. SantaLucia, H.T. Allawi and P.A. Seneviratne, Biochemistry 35, 3555-3562 (1996).

18. J. Marmur and P. Doty, J. Mol. Biol. 5,109-118 (1962).

19. M.J. Lane, T. Paner, I. Kashin, B.D. Faldasz, B. Li, F.J. Gallo and A.S. Benight, Nucleic Acids Res. 25, 611-616 (1997).

20. R. Elghanian, J.J. Storhoff, R.C. Mucic, R.L. Letsinger and C.A. Mirkin, Science 277, 1078-1081 (1997).

21. J.J. Storhoff, R. Elghanian, R.C. Mucic, C.A. Mirkin and R.L. Letsinger, J. Am. Chem. Soc. 120, 1959-1964 (1998).

22. T.A. Taton, C.A. Mirkin and R.L. Letsinger, Science, 289, 1757-1760 (2000).

23. T.A. Taton, G. Lu and C.A. Mirkin, J. Am. Chem. Soc., 123, 5164-5165 (2001).

24. T.M. Paner, F.J. Gallo, M.J. Doktycz and A.S. Benight, “Biopolymers 33, 1779-1789 (1993).

25. N. Sugimoto, S. Nakano, M. Yoneyama and K. Honda, Nucleic Acids Res. 24, 45014505 (1996).

26. S.G. Lokhov and D.V. Pyshnyi, FEBS Letters 420, 134-138 (1997).

27. M. Senior, R.A. Jones and K.J. Bresaluer, Biochemistry 27, 3879-3885 (1988).

28. A. Lane, S.R. Martin, S. Ebel and T. Brown, Biochemistry 31, 12087-12095 (1992). 
29. T. Ohmichi, S. Nakano, D. Miyoshi and N. Sugimoto, J. Am. Chem. Soc. 124, 10367$10372(2002)$. 
(a) Nick
3'-ACC ACC ACG GCG CTT GAG ATA AAT-5'
5'-TGG TGG TGC CGC GAA CTC TAT TTA-3'
X 3 , 5, $\quad$ Y

(c) Nick/mismatch

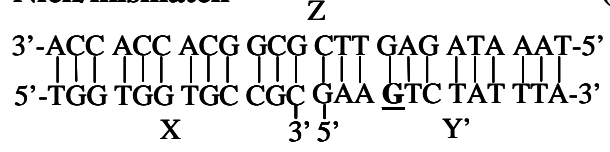

(b) Gap

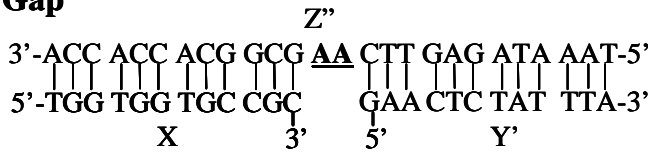

(d) Gap/mismatch

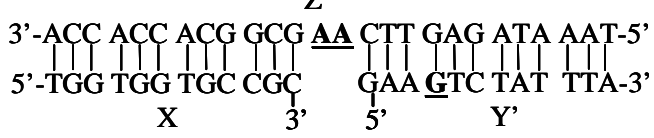

Figure 1 Base sequences of the four oligonucleotide complexes used in the study, i.e., nick (XZY), gap (XZ'YY), nick/mismatch (XZY') and gap/mismatch (XZ''Y'). Positions of each gap and mismatch are underlined. 


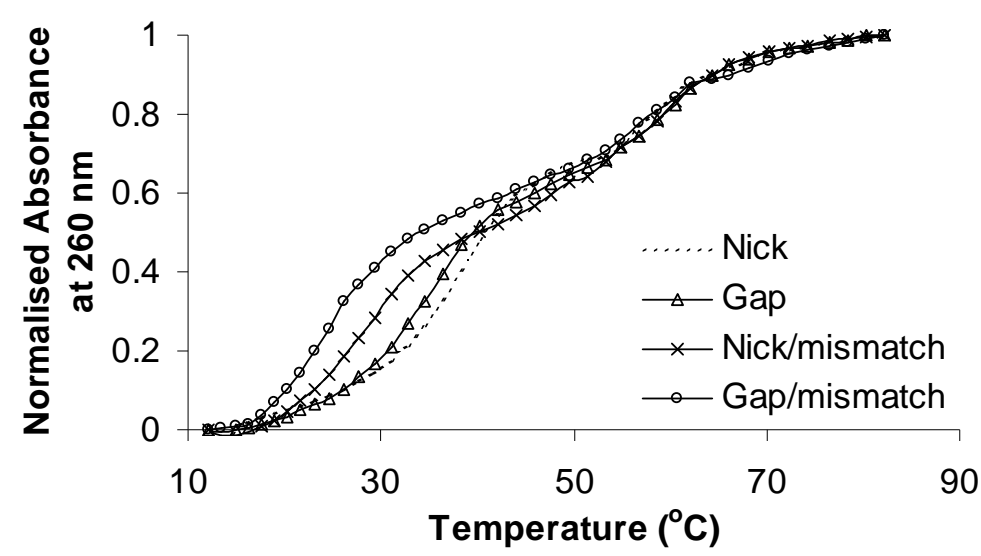

Figure 2 Normalised absorbance versus temperature melting profiles collected for the nick (XZY), gap (XZ''Y), nick/mismatch (XZY') and gap/mismatch (XZ'’Y') complexes. 


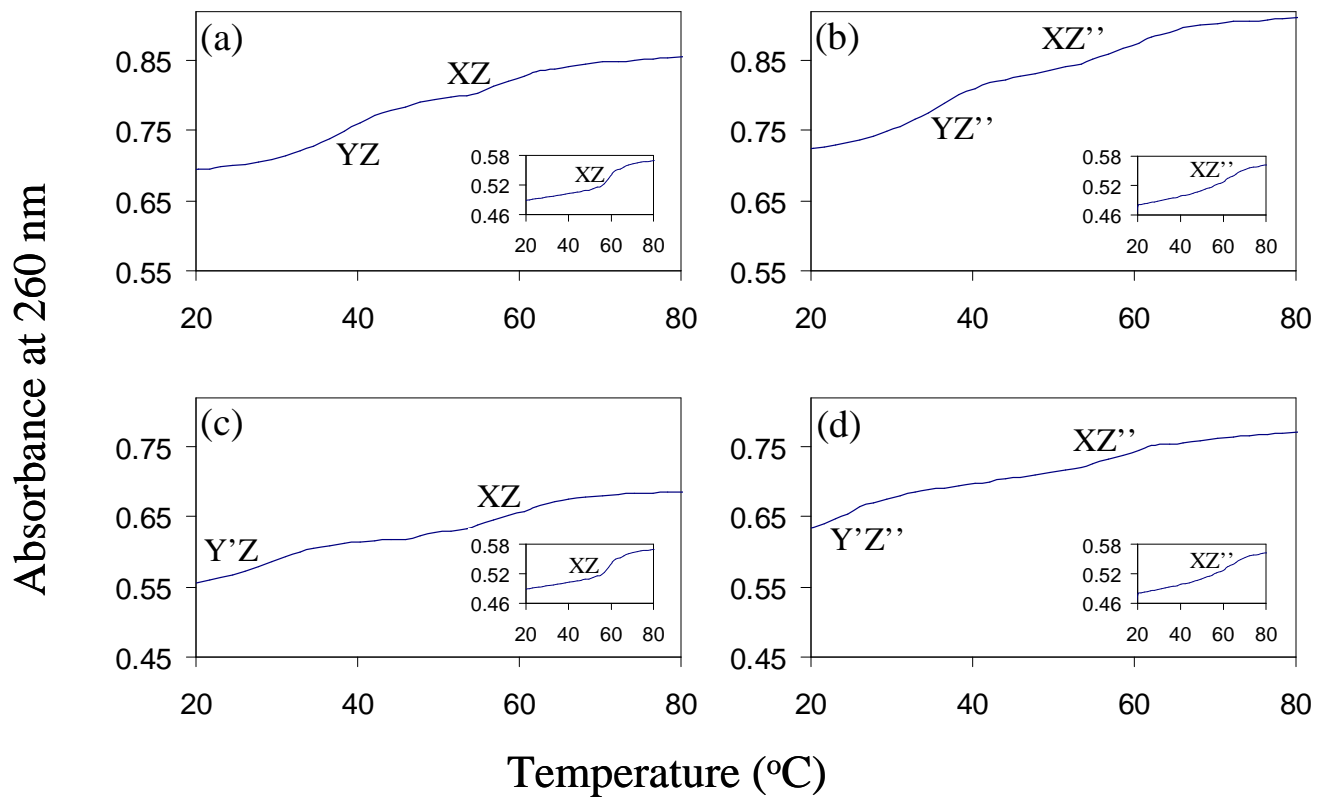

Figure 3 Melting curves for the each of the investigated complexes: (a) nick (XZY), (b) gap (XZ''Y), (c) nick/mismatch (XZY') and (d) gap/mismatch (XZ''Y') (data of Figure 2). The insets show the melting profiles for the corresponding $\mathrm{XZ}$ and $\mathrm{XZ}$ '' duplexes alone. 


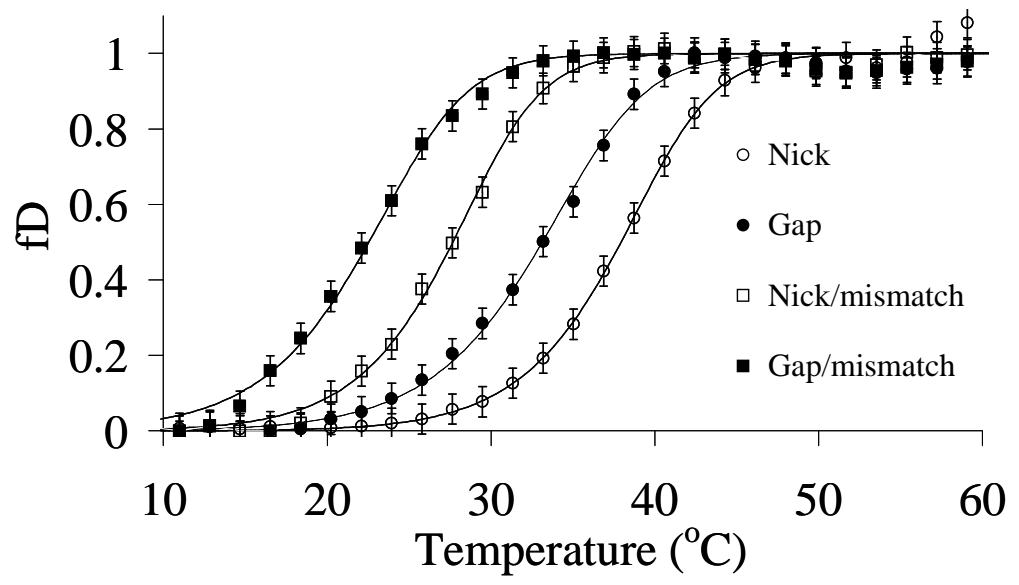

Figure 4 Plot of experimentally derived $f_{D}$ versus temperature data for each of the complexes, where $\mathrm{f}_{\mathrm{D}}$ is the fraction of each $\mathrm{Y}$ or $\mathrm{Y}^{\prime}$ strand that has dissociated from each complex, are shown using symbols. Fits to the data using Equation 6 are shown using solid lines. 
Table I Extracted thermodynamic parameters for the dissociation of $12 \mathrm{mer} \mathrm{Y}$ or Y' strands from each of the three-strand complexes. For comparison, thermodynamic parameters calculated for the $12 \mathrm{mer}$ Y sequence and its complementary 12 mer using nearest neighbour methods of (a) Sugimoto et al. (21) and (b) SantaLucia et al. (17) are also included.

\begin{tabular}{|c|c|c|c|}
\hline Complex & $\begin{array}{c}\Delta \mathbf{H}^{\circ} \\
(\mathbf{k c a l} / \mathbf{m o l})\end{array}$ & $\begin{array}{c}\Delta \mathbf{S}^{\circ} \\
(\mathbf{c a l} / \mathbf{m o l K})\end{array}$ & $\begin{array}{c}\Delta \mathbf{G}^{\circ} \\
(\mathbf{k c a l} / \mathbf{m o l})\end{array}$ \\
\hline Nick (XZY) & -94.5 & 276.5 & -13.5 \\
\hline Gap (XZ'’Y) & -82.9 & 243.3 & -11.6 \\
\hline Nick/mismatch (XZY') & -93.0 & 282.0 & -10.4 \\
\hline Gap/mismatch (XZ'Y') & -81.3 & 247.5 & -8.8 \\
\hline Y/complement calculated ${ }^{(a)}$ & -87.8 & 249.4 & -14.7 \\
\hline Y/complement calculated ${ }^{(b)}$ & -83.1 & 236.4 & -13.8 \\
\hline
\end{tabular}

